\title{
Near-IR spectroscopic monitoring of WR 140 (WC7pd+O4-5) during the 2001 periastron passage
}

\author{
Watson P. Varricatt ${ }^{1}$, Peredur M. Williams ${ }^{2}$, and Nagarhalli M. Ashok ${ }^{3}$ \\ ${ }^{1}$ Joint Astronomy Centre, Hawai'i Headquarters, University Park, \\ 660 North A'ohoku Place, Hilo, HI 96720, USA \\ ${ }^{2}$ Institute for Astronomy, University of Edinburgh, Royal Observatory, \\ Blackford Hill, Edinburgh EH9 $3 H J$, Scotland \\ ${ }^{3}$ Astronomy and Astrophysics Division, Physical Research Laboratory, \\ Navrangpura, Ahmedabad, 380009 India
}

\begin{abstract}
The near-IR spectrum of the periodic dust making WCpd+O4-5 binary WR 140 was monitored to cover the 2001 periastron passage and maximum colliding-wind activity. The He I $\lambda 1.083 \mu \mathrm{m}$ emission-line profile showed the appearence of a subpeak on epochs close to periastron passage. The evolution of the subpeak was consistent with the motion of the stars and the colliding wind region. The appearance and evolution of the emission subpeak suggests that the theoretical $1 / r$ dependence of X-ray flux does not hold, so that there is no need to change the values of eccentricity and epoch of periastron passage derived from the RV orbit. JHK spectra show variations of the continuum and and dilution of the emission lines, in agreement with the production and cooling of dust.
\end{abstract}

\section{Introduction and observations}

WR 140 (HD 193793, WC7+O4-5, $P=7.94 \mathrm{yr}, e=0.84$ ) is the archetype of the known WC episodic dust forming colliding-wind binaries (Williams et al. 1990; Williams 1995), which also shows variable X-ray and non-thermal radio emissison. Dust formation occurs for only during a tiny fraction (3-4\%) of the orbital period, around periastron passage, when the colliding winds of the two stars are compressed (Williams et al. 1990). The spectrum of WR 140 was monitored in the JHK-bands in March 2001, using the UKIRT-CGS4 at a resolution $R=800$ ( $J$-band) and $R=400$ ( $H$ and $K)$. In 2001 January, a $J$ spectrum at $R=1000$ was observed using the $1.2 \mathrm{~m} \mathrm{Mt}$. Abu Telescope (India). The He I $\lambda 1.083 \mu \mathrm{m}$ emission line, which is a good tracer of colliding wind phenomena (Stevens \& Howarth 1999), was observed from 2000 October to 2001 March, thus covering the periastron, using CGS4 at $R=4700$.

\section{Results and discussions}

The continua of the $J$-band spectra taken in 2001 January and 2002 April are similar in slope and the lines have similar equivalent widths $\left(W_{\lambda}\right)$, whereas the $J$ spectrum of 2001 March 31 is much flatter and the lines have smaller $W_{\lambda}$. This is due to varying amount of dilution by thermal emission from the dust. This also implies, that the dust formation started at an epoch between early January and late March 2001. By April 2002, the dust had cooled sufficiently for the $J$ spectrum not to be affected. A similar effect is shown in the $H$ and $K$-band 

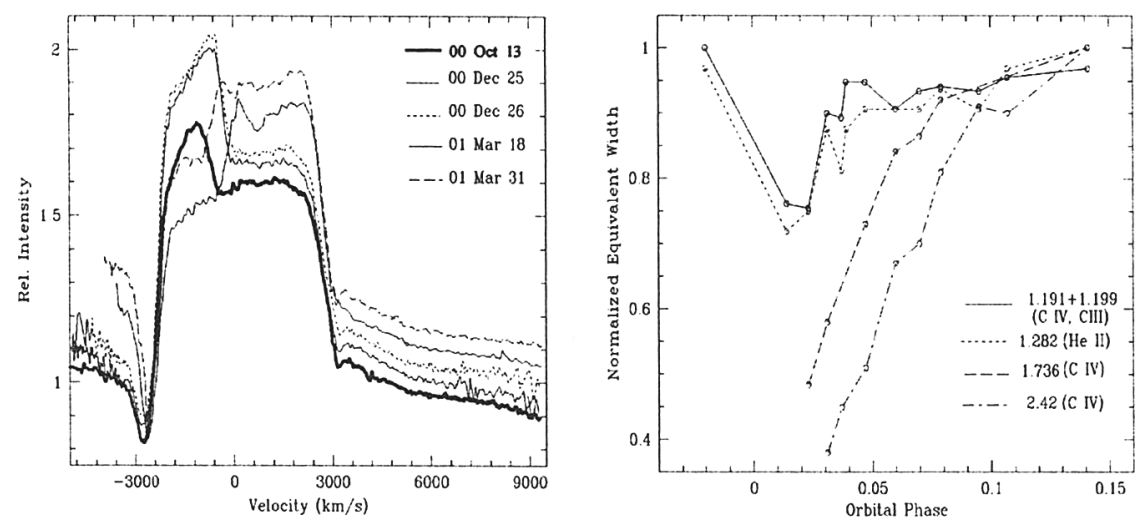

Figure 1. Evolution of He I $\lambda 1.083 \mu \mathrm{m}$ profile and $W_{\lambda}$ of WR 140.

spectra. The $W_{\lambda}$ of four emission lines, normalized to the values away from periastron, are plotted in Figure $1 \mathrm{~b}$ at different phases. The phase lag in the rise of $W_{\lambda}$ with wavelength is due to the cooling of the dust and the subsequent shift of the peak dust emission to longer wavelengths.

Figure 1a shows the change in the line profile of $\mathrm{He}$ I $\lambda 1.083 \mu \mathrm{m}$ during periastron passage. The subpeak is formed on the bow shock between the two winds. The HeI line profiles of the spectra taken before 2001 February show the subpeak blue shifted. At these epochs, the O-type component was in front, and material flowing along the shock cone had a net velocity towards the observer. The intensity of the subpeak also increased from October to December 2000 , when the stars moved closer to each other. The spectra from March 2001 show the subpeak red shifted. These are epochs after the periastron passage, when the shock cone was tilted away from the observer. The red-shifted subpeak emission from the colliding wind region appears to suffer absorption by the WR wind during these epochs. The absorption was greater when the stars were closer. Consequently, the subpeak on March 18 was fainter than that on March 31. The subpeak had not been previously observed on the He I line in WR 140, but has been observed from other, closer, WR+O binaries (Stevens \& Howarth 1999). It is likely to be a strong coolant of the shock-heated gas, aiding dust formation and and also influencing the $\mathrm{X}$-ray emission. The appearance of the subpeak around periastron passage implies, that the wind was probably not adiabatic then, so that the theoretical $1 / r$ dependence of the X-ray flux does not hold, and there is no need to adjust the orbital parameters to fit the X-ray data (Zhekov \& Skinner 2000; Setia Gunawan et al. 2001).

\section{References}

Setia Gunawan, D.Y.A., van der Hucht, K.A., Williams, P.M., Henrichs, H.F., Kaper, L., Stickland, D.J., Wamsteker, W. 2001, A\&A 376, 460

Stevens, I.R., Howarth, I.D. 1999, MNRAS 302, 549

Williams, P.M., van der Hucht, K.A., Pollock, A.M.T., et al. 1990, MNRAS 243, 662

Williams, P.M. 1995, in: K.A. van der Hucht \& P.M. Williams (eds.), Wolf Rayet Stars: Binaries, Colliding Winds, Evolution, Proc. IAU Symp. No. 163 (Dordrecht: Kluwer), p. 335

Zhekov, S.A., Skinner, S.L. 2000, ApJ 538, 808 